\title{
System-level Prognostics based on Inoperability Input-output Model
}

\author{
Ferhat Tamssaouet ${ }^{1}$, TP Khanh Nguyen ${ }^{2}$, and Kamal Medjaher ${ }^{3}$ \\ ${ }^{1,2,3}$ Laboratoire Génie de Production, LGP, Université de Toulouse, INP-ENIT, 47 Av. d'Azereix, 65016, Tarbes, France \\ ferhat.tamssaouet@enit.fr \\ thi-phuong-khanh.nguyen@enit.fr \\ kamal.medjaher@enit.fr
}

\begin{abstract}
Nowadays, the modern industry is increasingly demanding the availability and reliability of production systems as well as the reduction of maintenance costs. The techniques to achieving these goals are recognized and discussed under the term of Prognostics and Health Management (PHM). However, the prognostics is often approached from a component point of view. The system-level prognostics (SLP), taking into account interdependencies and multiinteractions between system components, is still an underexplored area. Inspired from the inoperability inputoutput model (IIM), a new approach for SLP is proposed in this paper. The inoperability corresponds to the component's degradation, i.e. the reduction of its performance in comparison to an ideal reference state. The interactions between component degradation and the effect of the environment are included when estimating the inoperability of components and also when predicting the system remaining useful life (SRUL). This approach can be applied to complex systems involving multi-heterogeneous components with a reasonable computational effort. Thus, it allows overcoming the lack of scope and scalability of the traditional approaches used in PHM. An illustrative example is presented and discussed in the paper to highlight the performance of the proposed approach.
\end{abstract}

\section{INTRODUCTION}

The prognostics is the process of predicting the end of (useful) life (EOL) and/or the remaining useful life (RUL) of components, subsystems or systems. In the last two decades, the topic of prognostics has become a research framework in its own right with a profusion of papers covering a multitude of scientific problems and various fields of applications (Gouriveau, Medjaher, \& Zerhouni, 2016; Jardine, Lin, \& Banjevic, 2006). However, the prognostics has often been approached from a component view without considering

Ferhat Tamssaouet et al. This is an open-access article distributed under the terms of the Creative Commons Attribution 3.0 United States License, which permits unrestricted use, distribution, and reproduction in any medium, provided the original author and source are credited. interactions with the other system's components and the environment. In practice, it is not specifically components' life-times that are important, but rather, the lifetimes of the systems in which these components are located (Daigle, Bregon, \& Roychoudhury, 2012). For example, it is not the failure of a single pump that interests stakeholders in a manufacturing plant, but the unavailability of the delivered service. Therefore, it is necessary to study the prognostics term at system-level taking into account mutual interactions between components.

In detail, the SLP aims to estimate the RUL of a system (SRUL) knowing the state of health of its components as well as their interactions and future conditions of use. A system is defined here as a set of elements (components or subsystems) interacting with each other and with the environment in order to perform one or more tasks. The SRUL provides information related to the time when the whole system stops working (i.e., when the combined failures of individual components lead to a system failure) (Rodrigues, 2017). Therefore, SLP can bring benefits in all stages of the system life-cycle process (Sun, Zeng, Kang, \& Pecht, 2012) by increasing system reliability and availability, ensuring security and making systems more resilient. It allows identifying the crucial components to be monitored even if their RUL(s) are not low, but their influence on the degradation processes of other components are significant. On the other hand, the SLP approach can also improve the organization of the maintenance function: schedule systemwide maintenance, reduce the number of interventions, etc.

In general, the SLP approaches can be classified into two groups based on the point of view of the system modeling. The methods in the first group usually simplify the system modeling when evaluating the SRUL. In detail, the system can be considered as a black box, and the SRUL is estimated based on the input data using machine learning methods (Xiaochuan, Duan, Mba, \& Bennett, 2016). However, these methods require a lot of monitoring data that is not easy to acquire in practice. On the other hand, using risk analysis methods (for example, failure modes and effects analysis FMEA and preliminary risk analysis PRA) to identify the 
most critical component, the prognostics of system failure can be addressed by the component-level prognostics approaches (Brahimi, Medjaher, Leouatni, \& Zerhouni, 2016).

The second group aims to take into account multicomponent when evaluating the SRUL using data-driven or model-based approaches. In (Daigle, Bregon, \& Roychoudhury, 2012), the authors propose to decompose the physical model of a system into independent sub-models and then derive SRUL based on the RUL of components. However, this approach is based on the assumption that the subsystems are independent and cannot be widely used because of the analytic model complexity.

For the data-driven methods, Piecewise Deterministic Markov Process (PDMP) simulation was used in (Jie \& Zio, 2016) for evaluating the reliability and RUL of a system with two components. In (Kharasgani, Biswas, \& Sankararaman, 2017), a stochastic simulation was proposed to predict the SRUL and its uncertainty from the component RULs. Since the purpose of prognostics is to calculate the RUL, several methods have been proposed to determine it. For components in series, the SRUL represents the minimum RULs of the components whereas, for component in parallel, the SRUL corresponds to the maximum. Finally, other works related to SLP are proposed in (Desforges, Diévart, Charbonnaud, \& Archimède, 2012) and (Maitre, Gupta, Medjaher, \& Zerhouni, 2016), where functional and hardware redundancies were considered. However, all above studies are based on the assumption that the components are independent from each other and do not take into account the effect of the environment on the evolution of degradation.

In this context, the article aims to fill the gap presented in the literature. We propose a new approach based on the inoperability input-output model (IIM) to evaluate SRUL taking into account the mutual interactions between the components and the influence of the environment. The degradation of components is expressed in terms of inoperability, i.e. in relation to its performance, which allows us to consider heterogeneous components.

The remainder of the paper is organized as follows. In Section 2 , a description of the original IIM model is presented. A new SLP approach based on the dynamic IIM is introduced in Section 3, as well as the meaning of the various model's parameters. In Section 4, a numerical example is presented and discussed to highlight the performance of the proposed approach. Finally, the conclusion and perspective of this work are discussed in Section 5.

\section{BACKGROUND ON IIM}

IIM is inspired by the input-output model (I-OM) developed by Leontief Wassily in 1936 (Leontief, 1936) and for which he won the Nobel Prize in Economics in 1973. The I-O model focuses on inter-industry analysis to determine economic flows expressed as the quantity of goods traded between different sectors of activity. This model has been widely used in planned economies and is still used today in the national accounts of several countries in a form of input-output tables.

Then, the input-output inoperability model (IIM) (Haimes \& Jiang, 2001; Santos \& Haimes, 2004), was developed to express the global effects of negative events on highly interdependent infrastructures or multisector economy. It allows analyzing how a natural outage or attack on an infrastructure may affect other infrastructures, emphasizing the cascading effects and the intrinsic vulnerabilities. This dysfunctional model added consideration of physical flows between infrastructures in addition to economic flows.

IIM aims to the determination of the inoperability of a system after a negative event, which is defined as the inability of this system to perform its intended functions (percentage of the achievement of objectives). Inoperability can take different forms, depending on the nature of the problem and the type of the system. In circumstances where the level of production, if it is a major concern, it may well be defined as the unrealized production (i.e., the expected level of production minus the actual production) divided by the expected level of production. For example, if the system under consideration is a power plant, inoperability can be defined as the difference between the desired energy production level and the actual amount of energy produced divided by the desired production level.

In its static form, the IIM is presented under the following formula:

$$
q=A q+c
$$

where $q$ is the inoperability of sectors, $c$ is the inoperability brought by an external event (ex. drop in demand, industrial accident, etc.) and $A$ is the matrix of interdependencies.

The static model of IIM considers initial one-time perturbation, and does not take into account the evolution of the system after the perturbation. To fill this gap, a dynamic IIM is introduced. It considers the return to equilibrium of a system after a perturbation (Santos \& Haimes, 2004):

$$
\dot{q}(t)=K[A q(t)+c(t)-q(t)]
$$

with:

$$
\int_{0}^{+\infty} c(0) d t=\text { initial pertubation }
$$

$K$ represents the resilience factor (recovery rate), i.e. the capacity of an industry or infrastructure to return to its normal state before the disruption. This capacity may refer to redundancies on the infrastructures or the possibility of investing quickly in new equipment.

Various extensions of the model have been proposed in the literature. One can cite DIIM with varying time perturbation (Orsi \& Santos, 2010), which takes into account perturbations 
that do not occur once but vary over time (ex. the problem of absenteeism in the case of an epidemic). Within an uncertain context, the authors in (Oliva, Panzieri, \& Setola, 2011) propose to use fuzzy logic for the lack of statistical data.

\section{SYSTEM-LEVEL PROGNOSTICS BASED ON IIM}

The IIM is originally interested in modeling interconnected infrastructures in order to observe the effects of a perturbation and its propagation on the whole system. In SLP, however, we are considering systems consisting of several components with their own degradation processes and also interacting with each other. Therefore, each component can be considered as an infrastructure; and its influences on the degradation of others can be explained as the interactions between the infrastructures. The principal difference between the original model and our model is that in the case of risk analysis and economics, the model aims to find the equilibrium point, i.e. the state preceding the disruption. Whereas for prognostics, we focus on identifying the failure time of system, because this is the information that interests the practitioners.

The IIM model allows addressing the gaps identified in our review of the SLP literature. The notion of inoperability can be considered in several ways and this allows heterogeneous components with different degradation processes to be taken into account. The inoperability of a component can be caused by its own degradation (aging and wear) and an induced degradation due to the degradation of other components. The resilience factor in the IIM, which is renamed in this paper as the influence factor, makes it possible to take into account the effect of the environment on the evolution of the component's degradation.

In the following, the IIM approach for SLP and the parameter of the model will be presented.

\subsection{IIM formalization}

Let's consider a first order Markov process in order to capture the degradation processes over time. The proposed model based on IIM is used to evaluate the interoperability evolution of the system components with this recursive formula:

where:

$$
q(t)=K(t) \cdot[A \cdot q(t-1)+c(t)]
$$

- $q(t)$ is a vector representing the overall inoperability of the system's components;

- $A$ is a matrix representing the interdependencies between the system components;

- $c(t)$ represents the internal inoperability of system's components;

- $A q(t)$ represents the inoperability of a component due to its interdependencies;
- $K$ is a matrix representing the factors influencing the inoperability of the components.

The analytic solution of the model is given by the following expression:

$$
q(t)=e^{-K(I-A) t} q(0)+\int_{0}^{t} K e^{-K(I-A)(t-z)} c(z) d z
$$

This solution is valid when the interdependencies and the influence factors are constant over the time-life of the system.

\section{Remark:}

Currently, the state-space representation in control engineering is also widely applied for PHM (Sun, Zuo, Wang, \& Pecht, 2012). It is represented as follow:

$$
\dot{x}(t)=A x(t)+B u(t)
$$

where $x(t)$ is the state vector, $\dot{x}(t)$ is the rate of change of the state variable, $u(t)$ is the input vector, $A$ is the state (or system) matrix and $B$ is the input matrix.

The state space representation model (Eq. (6)) and the IIM (Eq. (4)) have some similarities, however, there are significant differences in their structure and the meaning of their parameters. Indeed, the state-space model is interested in the change of system states during the control loop while the IIM model focus on the degradation modeling of the system and its components. In detail, the IIM allows investigating the interactions between the system and its components or between the components. This allows the degradation model of one component (obtained empirically) to be reused in several systems provided that the interactions with the other components of these systems are known (i.e. the matrix $A$ ). Moreover, it also takes into account the influence of the operating conditions represented by the factor $K$. It is not direct part of the degradation model but a parameter that allows the evolution of the degradation to be modified. This will make it possible to determine a direct relationship between the use profile of a system and its degradation.

\subsection{Discussion of the model parameters}

\subsubsection{Inoperability}

It corresponds to a column vector of inoperabilities of the $n$ components of the system at time $t$ :

$$
q(t)=\left[q_{i}(t)\right] ; \quad \forall i=1,2, \ldots, n
$$

The inoperability $q_{i}(t)$ of a component represents the decrease in a component's performance compared to its flawless state (non-degraded performance). It is expressed as:

$$
q_{i}(t)=\frac{\mid\left(\text { performance }_{i}\left(t_{0}\right)-\text { performance }_{i}(t)\right) \mid}{\text { performance }_{i}\left(t_{0}\right)}
$$

The performance of a component is obtained by monitoring a health indicator (a sensor's measures) or a function combining several health indicators. 


\section{Properties:}

- The inoperability of each component is a unique value between 0 and 1 .

$\circ q_{i}(t)=0$ : the component is healthy (with the ideal performance);

○ $q_{i}(t)=1$ : the component is considered faulty, i.e. the component has reached the failure threshold.

- In general, at the initial state $t_{0}=0, q_{i}\left(t_{0}\right)=0$.

Without generality loss, the evolution of the inoperability of a component is an increasing function because the performance of a component naturally deteriorates over time. The RUL of component $i$-th is given by the difference between its failure time, $t_{f_{i}}$ where the inoperability will reach the failure threshold $\left(q_{i}(t)=1\right)$, and the current instant $t_{c}$.

$$
R U L_{i}=t_{f_{i}}-t_{c}
$$

In the case of components in series configuration, the SRUL is obtained as follow:

$$
S R U L=\min \left(R U L_{i}\right)
$$

whereas in parallel configuration:

$$
S R U L=\max \left(R U L_{i}\right)
$$

Transforming a health indicator to an inoperability value

Health indicators of components are used to monitor the evolution of its degradation. They are derived from physical parameters measured by sensors. However, these physical parameters vary over different intervals. That is why the values of the physical parameters must be normalized in a range of $[0,1]$, which will directly give us the inoperability.

For a health indicator whose values are increasing, the inoperability is obtained by:

where:

$$
q(t)=\frac{x(t)-x\left(t_{0}\right)}{L-x\left(t_{0}\right)}
$$

- $\quad x(t)$ is the value of the health indicator transmitted at time $t$ by the sensor;

- $x\left(t_{0}\right)$ is the value of the health indicator at the initial time $t_{0}$;

- $\quad L$ is the failure threshold of the health indicator.

If the values returned by a sensor are decreasing, then the normalization is done as follows:

$$
q(t)=\frac{x\left(t_{0}\right)-x(t)}{x\left(t_{0}\right)-L}
$$

This method is well suited to normalization because 1 ) it does not introduce distortion into the data; and 2) presents a direct relationship between the data before and after transformation.
However, it assumes that all input values of the sensors belong to a determined interval known a priori If there is a new data that goes outside the interval already set, the model will be distorted. This problem can be addressed by applying out-of-range methods, which make it possible not to consider the values outside of the determined intervals, or to consider them equal to 1 (if $x(t)>L$ ) or 0 (if $x(t)<x\left(t_{0}\right)$ ). On the other hand, the normalization method based on the logistic function (sigmoid function) can be used (Jayalakshmi \& Santhakumaran, 2011). This function transforms all $\mathbb{R}$ values of the health indicator into values going from 0 to 1 . However, it is necessary to transform the raw data $x$ in $x^{\prime}$ in order to obtain symmetrical values of the health indicator:

$$
x^{\prime}=\frac{(x-\tilde{x})}{\lambda \frac{\delta}{2 \pi}}
$$

where: $\tilde{x}$ is the mean value, $\lambda$ is the size of interval in which we want to normalize (which is equal to 1 in this case) and $\delta$ is the standard deviation.

Thus, the normalized value can be obtained by using the logistic function:

$$
y=\frac{1}{1+e^{-x \prime}}
$$

For example, let's consider a component whose health indicator $x(t)$ increase linearly from 0 to 100 . Using the Eq. (14), the transformed values of the health indicator $x^{\prime}(t)$ will increase from -10.88 to 10.88 . Finally, using the Eq. (15), the normalized value $y(t)$ obtained belongs to the range $\left[e^{-5}, 0.99\right]$. So, it can be seen that this method ensures that the inoperability interval $[0,1]$ is not exceeded, but it involves distortion in the inputs and therefore lead to loss information about the nature of the degradation process. Another disadvantage of the logistics function is that the values 0 and 1 are never reached, therefore the initial condition as well as the threshold must be redefined, for example approximately equal to 0 or 1 .

In summary, both above normalization methods allow addressing two problems of the SLP 1) different health indicators with heterogeneous intervals, and 2) different failure threshold for homogeneous components (with the same health indicators).

\subsubsection{Matrix of interdependencies}

This matrix probes the different interdependencies between the system's components.

$$
A=\left[a_{i j}\right] ; \quad \forall i, j=1,2, \ldots, n
$$

Each component $a_{i j}$ of the matrix corresponds to the influence of the inoperability of component $j$ on the inoperability of component $i$, i.e. the inoperability brought by component $j$ to component $i$. 


\section{Properties:}

$$
a_{i j}=\frac{d q_{i}}{d q_{j}}
$$

- $A$ is a square matrix $(N \times N$ where $N$ is number of components);

- When $i=j, a_{i j}=0$ because it is considered that the inoperability of a component does not affect the component itself;

- The bigger $a_{i j}$ is, the greater is the influence of $j$ on $i$.

\subsubsection{Matrix of influence factors}

As all systems interact with their environment, it is necessary to take into account the environmental conditions when considering the evolution of the system state. These conditions consist of environmental parameters (ambient temperature, humidity, etc.) or operating conditions (settings, production loads, etc.) and affect the system during the major phases of the life cycle. In our model, the effect of the environment is represented by the parameter $K$ :

$$
K=\operatorname{diag}\left[k_{i}\right]
$$

with $k_{i}$ is specific to each component and its assumed to be constant in time under the same environmental conditions.

Without generality loss, $k_{i} \geq 0$. The signification of different values of $K$ is explained in Table 1.

Table 1: Signification of the influence factor $K$

\begin{tabular}{|c|l|l|}
\cline { 2 - 3 } \multicolumn{1}{c|}{} & Inoperability & Meaning \\
\hline$k_{i}=0$ & $q_{i}$ stationary & $\begin{array}{l}\text { The component does not } \\
\text { degrade }\end{array}$ \\
\hline$k_{i}>0$ & $\begin{array}{l}-k_{i}=1: \text { normal case when } \\
\text { system operates in a normal } \\
\text { condition with a normal work } \\
\text { load; } \\
-0<k_{i}<1 \text { : when system } \\
\text { operates in a favorable } \\
\text { environment or with a low } \\
\text { work load, its degradation } \\
\text { processes is slower than the } \\
\text { normal case; } \\
-q_{i}>1 \quad \text { accelerated } \\
\text { degradation due to a hostile } \\
\text { environment or a high work } \\
\text { load. }\end{array}$ \\
\hline
\end{tabular}

\section{APPLICATION EXAMPLE}

In this section, a numerical example is presented to illustrate the application of the proposed model on SLP.

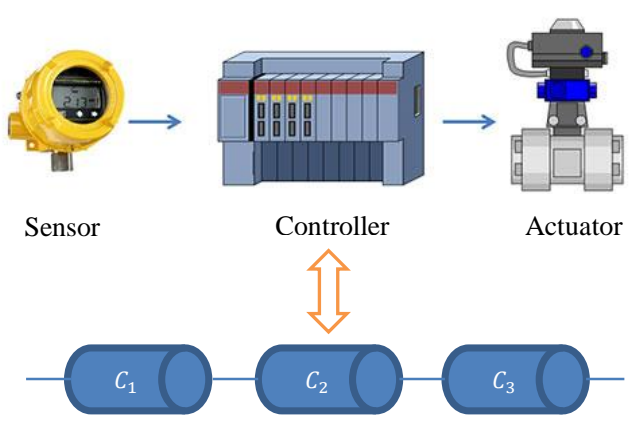

Figure 1: System composed of three components in series

Consider a control loop consisting of a sensor, a controller and an actuator connected in series. This control loop is intended to monitor the operating parameters of a system, and to ensure that they are permanently maintained within a determined range. The components of this control loop are subject to degradation, that reduces their performance, and which correspond to the following models, (resp. for the sensor $c_{1}$, the controller $c_{2}$ and the actuator $c_{3}$ ):

$$
\begin{gathered}
c_{1}(t)=0.7 \frac{t}{2} \\
c_{2}(t)=\frac{t}{10} \ln (t+1) \\
c_{3}(t)=e^{\frac{t}{40}}
\end{gathered}
$$

In this example, we assume that the degradation of the system is not influenced by external factors, i.e.:

$$
k_{i}=1 \quad, \quad \forall i=1,2,3
$$

The matrix of interdependencies is as follows:

$$
A=\left[\begin{array}{ccc}
0 & 0.02 & 0.09 \\
0.07 & 0 & 0.1 \\
0.08 & 0.2 & 0
\end{array}\right]
$$

That means, for $a_{12}$, at each time, $2 \%$ of the inoperability of the components 2 (the controller) will be transmitted to the component 1 (the sensor).

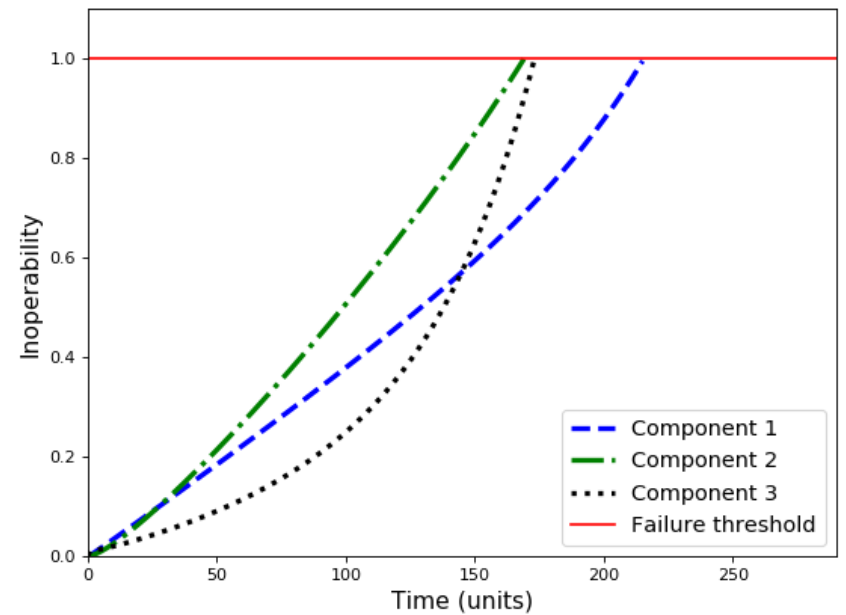

Figure 2: Components' inoperability evolution 
The evolution of the degradation of the components of the control loop is presented in Figure 2.

As component 2 (the controller) is the first failed component, the SRUL corresponds to its RUL and is equal to 168 time units.

From figure 2, one can notice that when $t<145$, the inoperability of component 3 is lower than the one of component 1 . When $t>145$, the opposite conclusion is occurring. This confirms the advantage of SLP over prognostics at component level, since at one point a most critical component may become less critical an instant later.

\section{Variation of influence factors}

Figure 3 presents the variation of the remaining useful life of the components (figure 3.a) and the system (figure 3.b) due to the environment effect by considering different values of the influence factors $k_{i}$.

The obtained results (figure 3 ) shows that when $k_{i} \rightarrow 0$, the RULs of the components and the SRUL tend towards $+\infty$. This result is predictable, because when $k_{i}=0$, the component is considered not to degrade and can operate indefinitely without reaching the failure threshold.

In the interval $[0.08,1.75]$, the SRUL decreases slightly, i.e. it is operating conditions that do not stress it. In fact, this

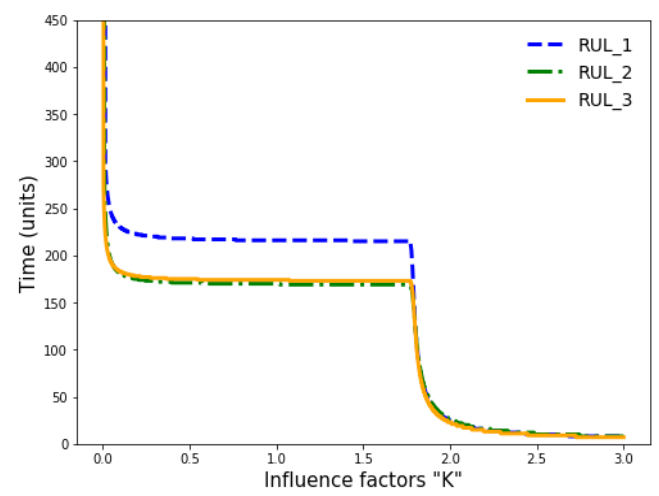

(a)

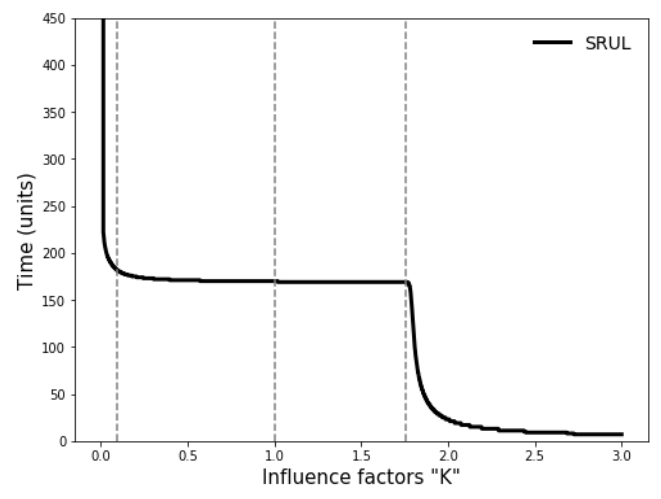

(b)

Figure 3: Variation of RULs (a) and SRUL (b) as a function of $K$ result can be explained when the system operates in a favorable environment thanks to good ambient conditions, good lubrication, a lower workload, etc. The environment accelerates the degradation of the system but in a nonsignificant way for $k_{i}$ in $[1,1.75]$.

Next, the system is considered stressed when $k_{i}>1.75$. In this case, the SRUL decreases rapidly. By knowing the relationship between environmental conditions or operating conditions and the evolution of component degradation, we can determine which environments are bearable and the workloads that the components can support.

\section{Conclusion}

In this paper, a new approach for system-level prognostics based on inoperability input-output model is proposed. The first contribution of this model is to take into account the interactions between the components to determine the SRUL more precisely. Initially, these interactions were considered linear, i.e. the ratio between the degradation of one component and those induced in the other components is constant at all times.

The second contribution concerns the consideration of the effect of the environment in the evolution of component degradation through a parameter of the model called: the influence factor. It allows providing more information about the environment conditions in which the system can operate without accelerating degradation.

This work is a first attempt to resolve the various locks of the prognostics of systems. Several perspectives are considered when using the IIM model. Given the complexity and timedependence of the interactions between the components, interdependence matrices with variable components can be considered and inoperabilities in the form of probabilities can be introduced to take into account the stochastic nature of the degradations.

\section{REFERENCES}

Brahimi, M., Medjaher, K., Leouatni, M., \& Zerhouni, N. (2016). Critical components selection of a prognostics and health management system design: an application to an overhead contact system. Annual Conference of the Prognostics and Health Management Society, (pp. 1-8). Colorado.

Daigle, M., Bregon, A., \& Roychoudhury, I. (2012). A Distributed Approach to System-Level Prognostics. Annual conference of the prognostics and health management society, (pp. 71-82).

Desforges, X., Diévart, M., Charbonnaud, P., \& Archimède, B. (2012). A Distributed Architecture to implement a Prognostic Function for Complex Systems. First European Conference of the Prognostics and Health Management Society, (pp. 2-9). Dresden. 
Gouriveau, R., Medjaher, K., \& Zerhouni, N. (2016). From Prognostics and Health Systems Management to Predictive Maintenance 1: Monitoring and Prognostics (1 Edition ed.). Wiley-ISTE.

Haimes, Y. Y., \& Jiang, P. (2001). Leontief-based Model of Risk in Complex Interconnected Infrastructures. Journal of infrastructure systems, 7(1), 1-12.

Jardine, A. K., Lin, D., \& Banjevic, D. (2006). A Review on Machinery Diagnostics and Prognostics implementing Condition-based Maintenance. Mechanical Systems and Signal Processing, 20(7), 1483-1510.

Jayalakshmi , T., \& Santhakumaran, A. (2011). Staistical Normalization and Back Propagation for Classification. International Journal of Computer Theory and Engineering, 3(1), 89-93.

Jie, L., \& Zio, E. (2016). Dynmaic reliability assessment and prognostics with monitored data for multiple dependent degradation components. European Safety and Reliability Conference. Glasgow.

Kharasgani, H., Biswas, G., \& Sankararaman, S. (2017). Methodologies for System-Level Remaining Useful Life Prediction. Reliability Engineering \& System Safety, 154(Suplement C), 8-18.

Leontief, W. W. (1936). Qunatitative Input and Output Relations in the Economic Systems of the United States. The review of economic statistics, 105-125.

Maitre, J., Gupta, J. S., Medjaher, K., \& Zerhouni, N. (2016). A PHM system approach: Application to a simplified aircraft bleed system. (IEEE, Ed.) Aerospace Conference, 2016 IEEE, pp. 1-9.

Oliva, G., Panzieri, S., \& Setola, R. (2011). Fuzzy Dynamic Input-output Inoperability Model. International Journal of Critical Infrastructure Protection, 4(3-4), 165-175.

Orsi, M. J., \& Santos, J. R. (2010). Incorporating TimeVarying Perturbations into the Dynamic Inoperability Input-output Model. IEEE Transactions on Systems, Man, and Cybernetics-Part A: Systems and Humans, 40(1), 100-106.

Rodrigues, L. (2017). Remaining Useful Life Prediction for Multiple-Component Systems Based on a System-Level Performance Indicator. IEEE/ASME Transactions on Mechatronics, 32(1), 141-150.

Santos, J. R., \& Haimes, Y. Y. (2004). Modeling the Demand Reduction Input-Output (I-O) Inoperability Due to Terrorism of Interconnected Infrastructures. Risk analysis, 24(6), 1437-1451.

Sun, B., Zeng, S., Kang, R., \& Pecht, M. G. (2012). Benefits and Challenges of System Prognostics. IEEE Transactions on reliability, 61(2), 323-335.

Sun, J., Zuo, H., Wang, W., \& Pecht, M. G. (2012). Application of a State Space Modeling Technique to System Prognostics Based on a Health Index for Condition-Based Maintenance. Mechanical Systems and Signal Processing, 28, 585-596.
Xiaochuan, L., Duan, F., Mba, D., \& Bennett, I. (2016). Rotating Machine Prognostics Using System-Level Models. In Engineering Asset Management (pp. 123141). Lecture Notes in Mechanical Engineering.

\section{BIOGRAPHIES}

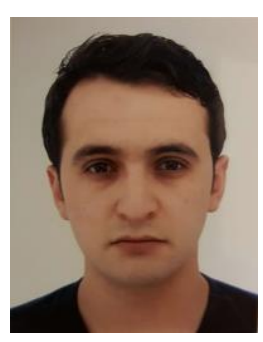

Ferhat Tamssaouet received the Engineering degree in industrial risk management from National Polytechnic School of Algiers, Algeria, in 2016, and the MS degree in complex systems engineering from the Ecole Normale Supérieure Paris-Saclay, Cachan, France, in 2017. Now, he is a PhD student at Laboratoire Génie de Production, INPENIT, Tarbes, France. His research interests include dependability, discrete event systems and system-level prognostics.

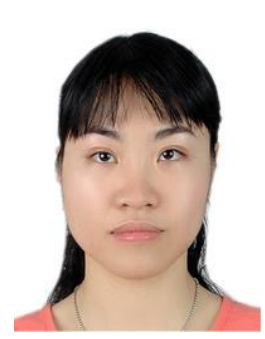

Khanh T.P. Nguyen received the Ph.D. degree in Automation and Production Engineering from École Centrale de Nantes, France in 2012. From 2013 to 2015, she was a Postdoctoral Fellow at the French Institute of Science and Technology for Transport, Development and Networks (IFSTTAR). From 2016 to 2017, she was an Assistant Professor at the University of Technology of Troyes. In September 2017, she joined the École Nationale d'Ingénieurs de Tarbes (ENIT), Toulouse INP as an Associate Professor. Her research interests include prognostics health management, maintenance optimization, and safety assessment of railway safety related systems.

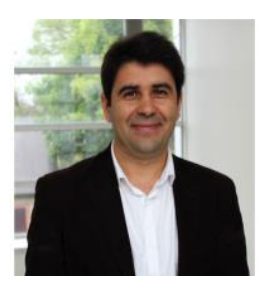

Kamal Medjaher received his BS degree in electronics, his MS in control and industrial computing in 2002 at the Ecole Centrale de Lille, and his $\mathrm{PhD}$ in 2005 in the same field from the University of Lille 1. Since February 2016, he is Full Professor at the Tarbes National School of Engineering (ENIT), France. He conducts his research activities within the Production Engineering Laboratory (LGP). Before this position, he was Associate Professor at the National Institute of Mechanics and Microtechnologies in Besançon (ENSMM), France, from September 2006 to January 2016.

Since September 2006, Prof. Medjaher leads research works in the field of prognostics and the health management of industrial systems. 